\title{
ELLIPTIC GW INVARIANTS OF BLOWUPS ALONG CURVES AND SURFACES
}

\author{
JIANXUN HU AND HOU-YANG ZHANG
}

Received 27 January 2004

We established a relation between elliptic Gromov-Witten invariants of a symplectic manifold $M$ and its blowups along smooth curves and surfaces.

\section{Introduction}

Over the last few years, many mathematicians contributed their efforts to establish the mathematical foundation of the theory of quantum cohomology or Gromov-Witten (GW) invariants. In 1995, Ruan and Tian [13, 15] first established for the semipositive symplectic manifolds. Recently, the semipositivity condition has been removed by many authors. Now, the focus turned to the calculations and applications. Many Fano manifolds were computed. We think that it is important to study the change of GW invariants under surgery.

Some recent research indicated that there is a deep amazing relation between quantum cohomology and birational geometry. The quantum minimal model conjecture [14] leads to attempt to find quantum cohomology of a minimal model without knowing minimal model. This problem requires a thorough understanding of blowup type formula of GW invariants and quantum cohomology.

According to McDuff [11], the blowup operation in symplectic geometry amounts to a removal of an open symplectic ball followed by a collapse of some boundary directions. Lerman [8] gave a generalization of blowup construction, "the symplectic cut."

Let $M$ be a compact symplectic manifold of dimension $2 n, \tilde{M}$ the blowup of $M$ along a smooth submanifold. Denote by $p: \tilde{M} \rightarrow M$ the natural projection. Denote by $\Psi_{(A, g)}^{M}\left(\alpha_{1}\right.$, $\left.\ldots, \alpha_{m}\right)$ the genus $g \mathrm{GW}$ invariant of $M$. The authors refer the interested reader to [15] for the definition of GW invariants.

In $[5,6]$, we mainly concentrated on the changes of genus zero GW invariants under blowup along smooth curves and surfaces. In this paper, we mainly concentrate on the elliptic GW invariants. These invariants were first discussed in [1] and since then have been studied in various contexts, see [3, 12]. An elegant recursion was predicted in [2] using the method of Virasoro constraints. In this paper, we will generalize some results 
about the cases of blowing up along curves and surfaces to the case of genus-one. Our main results are the following.

Theorem 1.1. Suppose that $C$ is a smooth curve in $M$ such that either its genus $g_{0} \geq 2$ or $g_{0} \leq 1$ and $C_{1}(M)(C) \geq 0$, where $C_{1}(M)$ denotes the first Chern classes of $M . A \in H_{2}(M)$ such that $p !(A)=P D p^{*} P D(A)$ is a nonexceptional class in $H_{2}(\tilde{M}), \alpha_{i} \in H^{*}(M), 1 \leq i \leq m$ satisfy either $\operatorname{deg} \alpha_{i} \neq 1$ or $\operatorname{deg} \alpha_{i}=1$ and support away from $C$. Then,

$$
\Psi_{(A, 1)}^{M}\left(\alpha_{1}, \ldots, \alpha_{m}\right)=\Psi_{(p !(A), 1)}^{\tilde{M}}\left(p^{*} \alpha_{1}, \ldots, p^{*} \alpha_{m}\right) .
$$

About the changes of GW invariants of blow up of symplectic manifold along a smooth surface, in this paper, we will assume that the symplectic manifold is semipositive. Our result is the following theorem.

Theorem 1.2. Suppose that $M$ is a semipositive compact symplectic manifold and $S$ is a smooth surface in $M$. If $A \in H_{2}(M)$ such that $p !(A)=P D p^{*} P D(A)$ is a nonexceptional class in $H_{2}(\tilde{M}), \alpha_{i} \in H^{*}(M), 1 \leq i \leq m$, satisfy either $\operatorname{deg} \alpha_{i}>2$ or $\operatorname{deg} \alpha_{i} \leq 2$ and support away from $S$. Then, (1.1) holds.

\section{General review of gluing formula}

The proof of our result is an application of the gluing formula for symplectic cutting developed by Li and Ruan [9]. In algebraic geometry, Li [10] proved a completely analogous degeneration formula of GW invariants. Another symplectic version of the gluing formula of GW-invariants is due to Ionel and Parker [7].

Suppose that $H: M^{0} \rightarrow \mathbf{R}$ is a proper Hamiltonian function such that the Hamiltonian vector field $X_{H}$ generates a circle action, where $M^{0} \subset M$ is an open domain. By adding a constant, we may assume that 0 is a regular value. Then, $H^{-1}(0)$ is a smooth submanifold preserved by the circle action. The quotient $Z=H^{-1}(0) / S^{1}$ is the famous symplectic reduction. Namely, it has an induced symplectic structure. We can cut $M$ along $H^{-1}(0)$. Suppose that we obtain two disjoint components $M^{ \pm}$which have the boundary $H^{-1}(0)$. We can collapse the $S^{1}$-action on $H^{-1}(0)$ to obtain $\bar{M}^{ \pm}$containing a real codimension two submanifold $Z=H^{-1}(0) / S^{1}$, see [9, Section 2 and pages 156-157] for details. There is a map

$$
\pi: M \longrightarrow \bar{M}^{+} \cup_{Z} \bar{M}^{-}
$$

where $\bar{M}^{+} \cup_{Z} \bar{M}^{-}$is the union of $\bar{M}^{ \pm}$along $Z$.

To formulate the gluing formula, we need the terminology of relative GW invariants. Here, we copy the definition of [9, Section 4 and page 157]. We define a relative GW invariant $\Psi^{(M, Z)}$ by counting the number of relative stable holomorphic maps intersecting $Z$ at finitely many points with prescribed tangency. Let $T_{m}=\left(t_{1}, \ldots, t_{m}\right)$ be a set of nonnegative integers such that $\sum_{i} t_{i}=Z^{*}(A)$, where $Z^{*}$ is the Poincare dual of $Z$ and $A \in H_{2}(M)$. We order them such that $t_{1}=\cdots=t_{l}=0$ and $t_{i}>0$ for $i>l$. Consider the moduli space $M_{A}\left(g, T_{m}\right)$ of genus $g$ pseudoholomorphic maps $f$ such that $f$ has marked points $\left(x_{1}, \ldots, x_{m}\right)$ with the property that $f$ is tangent to $Z$ at $x_{i}$ with order $t_{i}$. Here, $t_{i}=0$ means that there is no intersection. Then, we compactify $\mathcal{M}_{A}\left(g, T_{m}\right)$ by $\bar{M}\left(g, T_{m}\right)$, 
the space of relative stable maps. We have evaluation map

$$
e_{i}: \bar{M}_{A}\left(g, T_{m}\right) \longrightarrow M
$$

for $i \leq l$ and

$$
e_{j}: \bar{M}_{A}\left(g, T_{m}\right) \longrightarrow Z
$$

for $j>l$. Roughly, the relative $\mathrm{GW}$-invariants are defined as

$$
\Psi_{\left(A, g, T_{m}\right)}^{(M, Z)}\left(\alpha_{1}, \ldots, \alpha_{l} ; \beta_{l+1}, \ldots, \beta_{m}\right)=\int_{\bar{M}_{A}\left(g, T_{m}\right)}^{v i r} \Pi_{i} e_{i}^{*} \alpha_{i} \wedge \Pi_{j} e_{j}^{*} \beta_{j} .
$$

Let $u=\left(u^{+}, u^{-}\right):\left(\Sigma^{+}, \Sigma^{-}\right) \rightarrow\left(M^{+}, M^{-}\right)$be $J$-holomorphic curves such that $u^{+}$and $u^{-}$ have $v$ ends and they converge to the same periodic orbits at each end. Suppose that $\Sigma^{ \pm}$ have $m^{ \pm}$marked points, respectively. Here, $\Sigma^{ \pm}$may not be connected, see [9, Section 4]. Suppose that $\Sigma=\Sigma^{+} \bigvee \Sigma^{-}$has genus $g$ and $[u(\Sigma)]=A$. If we consider the index of the operators $D_{u^{ \pm}}=D \partial_{J} u^{ \pm}$, see $[9$, Section 4$]$ for its definition, then we have the following proposition.

Proposition 2.1 (see [9, Theorem 5.1]).

$$
\text { Ind } D_{u^{+}}+\operatorname{Ind} D_{u^{-}}=2(n-1) v+2 C_{1}(A)+2(n-3)(1-g)+2 m \text {, }
$$

where $C_{1}$ is the first Chern class of $M$ and $m=m^{+}+m^{-}$.

Suppose that the homology classes of $u^{+}, u^{-}, u$ are $A^{+}, A^{-}, A$, respectively. If $\left(u^{+}, u^{-}\right)$is another representative and glue to $u^{\prime}$, by $\left[9\right.$, Lemma 2.11], we have $\left[u^{\prime}\right]=[u]$. Denote by $C=\left\{A^{+}, g^{+}, K^{+} ; A^{-}, g^{-}, K^{-}\right\}$the gluing component, where $K^{ \pm}=\left(0, \ldots, 0, k_{1}, \ldots, k_{v}\right)$ with $m^{ \pm}$zeros. The gluing formula of $\mathrm{Li}$ and Ruan counted the contribution of the gluing components to GW invariant of $M$. Denote by $\Psi_{C}$ the contribution of $C$.

Choose a homology basis $\left\{\beta_{b}\right\}$ of $H^{*}(Z, \mathbf{R})$. Let $\left(\delta_{a b}\right)$ be its intersection matrix. For the gluing component $C=\left\{A^{+}, g^{+}, K^{+} ; A^{-}, g^{-}, K^{-}\right\}$, we have the following gluing formula.

Proposition 2.2 (see [9, Theorem 5.8]). Let $\alpha_{i}^{ \pm}$be differential forms with $\operatorname{deg} \alpha_{i}^{+}=$ $\operatorname{deg} \alpha_{i}^{-}$even. Suppose that $\left.\alpha_{i}^{+}\right|_{Z}=\left.\alpha_{i}^{-}\right|_{Z}$ and hence $\alpha_{i}^{+} \cup_{Z} \alpha_{i}^{-} \in H^{*}\left(\bar{M}^{+} \cup_{Z} \bar{M}^{-} ; \mathbf{R}\right)$. Let $\alpha_{i}=$ $\pi^{*}\left(\alpha_{i}^{+} \cup_{Z} \alpha_{i}^{-}\right)$. The following gluing formula holds:

$$
\Psi_{C}\left(\alpha_{1}, \ldots, \alpha_{m^{+}+m^{-}}\right)=|K| \sum_{I, J} \Psi_{\left(A^{+}, g^{+}, K^{+}\right)}^{\left(\bar{M}^{+},\right.}\left(\alpha^{+}, \beta_{I}\right) \delta^{I, J} \Psi_{\left(A^{-}, g^{-}, K^{-}\right)}^{\left(\bar{M}^{-},\right.}\left(\alpha^{-}, \beta_{J}\right),
$$

where $\beta_{i} \delta^{i, j} \beta_{j}$ is associated to every intersection with $Z$ and $|K|=k_{1} \cdots k_{v}, \delta^{I, J}=\delta^{i, j} \cdots \delta^{i_{\nu}, j_{v}}$, $\Psi_{\left(A^{ \pm}, g^{ \pm}, K^{ \pm}\right)}^{\left(\bar{M}^{ \pm}, Z\right)}\left(\alpha^{ \pm}, \beta_{I}\right)$ denote the product of relative invariants corresponding to each component.

Proposition 2.3 (see [9, Remark 5.5]). For $C=\left\{A^{ \pm}, g^{ \pm},(0, \ldots, 0)\right\}$,

$$
\Psi_{C}\left(\alpha_{i}^{ \pm}\right)=\Psi_{\left(A^{ \pm}, g^{ \pm},(0, \ldots, 0)\right)}^{\left(\bar{M}^{ \pm}, Z\right)}\left(\alpha_{i}^{ \pm}\right) .
$$




\section{Proof of main theorem}

Proof of Theorem 1.1. Since $C$ is a smooth curve in $M$, the normal bundle $N_{C}$ is a symplectic vector bundle. By symplectic neighborhood theorem, there is a tubular neighborhood $\mathcal{N}_{\delta}(C)$ of $C$ which is symplectomorphic to the normal bundle $N_{C}$. We perform the symplectic cutting as in [5, Section 2.1]. We obtained

$$
\bar{M}^{+}=\mathbf{P}\left(N_{C} \oplus 0\right), \quad \bar{M}^{-}=\tilde{M} .
$$

From the divisor property, the skew symmetry of GW invariants and our assumptions, if we choose a sufficiently small $\delta>0$, without loss of generality, we may assume $\alpha_{i}^{+}=0$.

Similar to the proof of [5, Theorem 1.2], by the gluing formula of GW invariant, we first consider the contribution of each component to the GW invariants. Therefore, we consider the gluing component

$$
C=\left\{A^{+}, g^{+}, K^{+} ; A^{-}, g^{-}, K^{-}\right\},
$$

where $K^{ \pm}=\left(0, \ldots, 0, k_{1}, \ldots, k_{v}\right)$ with $m^{ \pm}$many zeros, respectively. From Proposition 2.1, we have

$$
\text { Ind } D_{u^{+}}+\operatorname{Ind} D_{u^{-}}=2(n-1) v+2 C_{1}(A)+2 m \text {, }
$$

since $g=1$ in our case.

We will use the same convention as [5]. We assume that $u^{ \pm}: \Sigma^{ \pm} \rightarrow M^{ \pm}$may have $l^{ \pm}$ connected components $u_{i}^{ \pm}: \Sigma_{i}^{ \pm} \rightarrow M^{ \pm}, i=1, \ldots, l^{ \pm}$. Suppose $\Sigma_{i}^{ \pm}$have arithmetic genus $g_{i}^{ \pm}, g^{ \pm}=\sum g_{i}^{ \pm}$, with $m_{i}^{ \pm}$marked points and $m^{ \pm}=\sum m_{i}^{ \pm}$. From [5, Remark 2.3], it is not difficult to see that $\bar{u}_{i}^{+}$can be identified as a stable $J$-holomorphic curve $h_{i}^{+}$in $\bar{M}^{+}$. Then, from [5, Proposition 2.4], we have

$$
\begin{aligned}
\operatorname{Ind} D_{u^{+}}= & \sum_{i=1}^{l^{+}} \operatorname{Ind} D_{u_{i}^{+}}=\sum_{i=1}^{l^{+}} \operatorname{Ind} D_{\bar{u}_{i}^{+}} \\
= & 2 \sum_{i=1}^{l^{+}} C_{1}\left[h_{i}^{+}\right]+2(n-3)\left(l^{+}-g^{+}\right) \\
& +2 v-2 \sum k_{i}+2 m^{+} .
\end{aligned}
$$

Now, we want to calculate $C_{1}\left[h_{i}^{+}\right]$in two cases of our theorem.

Case 1. The genus $g_{0} \geq 2$.

In this case, we claim that all stable $J$-holomorphic maps $h_{i}^{+}$can only stay in fibers of $\bar{M}^{+}=\mathbf{P}\left(N_{C} \oplus \mathcal{O}\right)$. Otherwise, suppose that there is a stable $J$-holomrphic curve $h_{i}^{+}$: $\Sigma \rightarrow \bar{M}^{+}$which does not stay in a fiber. Denote by $\pi: \mathbf{P}\left(N_{C} \oplus \mathcal{O}\right) \rightarrow C$ the projection of the projective bundle. Then, we have a stable $J$-holomorphic map $\pi \circ h_{i}^{+}: \Sigma \rightarrow C$ satisfying $\left[\pi \circ h_{i}^{+}\right] \neq 0$. We can perform pregluing as in [9, Section 5, pages 208-209] and obtain a system of small perturbed $J$-holomorphic curves $f_{n}: \Sigma_{n} \rightarrow C$ which represent the class $\left[\pi \circ h_{i}^{+}\right]$and satisfy the perturbed Cauchy-Riemann equation $\bar{\partial}_{J} f_{n}=\nu_{n}$, here 
$\Sigma_{n}$ is a smooth Riemann surface. Actually, we can choose $\nu_{n} \rightarrow 0$ as $n \rightarrow \infty$. Therefore, by Gromov compactness theorem, we have that $f_{n}$ weakly converges to a (possibly reducible) $J$-holomorphic curve $u=\left(u^{1}, \ldots, u^{N}\right)$ and $\left[\pi \circ h_{i}^{+}\right]=\sum_{j=1}^{N}\left[u^{j}\right] \neq 0$. Therefore, we have a nonconstant $J$-holomorphic curve $f: \Sigma_{1} \rightarrow C$ and $\Sigma_{1}$ has genus less than 2 . It is well known that if $f^{\prime}: S \rightarrow S^{\prime}$ is a holomorphic map between compact Riemann surfaces, then the genus of $S$ and $S^{\prime}$ satisfies $g(S) \geq g\left(S^{\prime}\right)$ unless $f^{\prime}$ is constant (see [4, page 219]). Since $g(C)=g_{0} \geq 2$, we have a contradiction. So, our claim is true.

A simple index calculation [5] shows that $C_{1}\left[h_{i}^{+}\right]=n \sum k_{j}$, where summation runs over ends of component $u_{i}^{+}$. In this case, we have

$$
\text { Ind } D_{u^{+}}=2(n-3)\left(l^{+}-g^{+}\right)+2(n-1) \sum k_{i}+2 v+2 m^{+} .
$$

Case 2. $g_{0} \leq 1$ and $C_{1}(M)(C) \geq 0$.

A simple calculation shows, that $C_{1}\left(\mathbf{P}\left(N_{C} \oplus \mathcal{O}\right)\right)=C_{1}(C)+C_{1}\left(N_{C}\right)+n \xi=C_{1}(M)+n \xi$, here $\xi$ is the class of infinite section in $\mathbf{P}\left(N_{C} \oplus \mathcal{O}\right)$ over $C$. Therefore, from our positive assumption, an intersection multiplicity calculation shows that

$$
\sum_{i=1}^{l^{+}} C_{1}\left[h_{i}^{+}\right] \geq n \sum k_{i} .
$$

In this case, we have

$$
\text { Ind } D_{u^{+}} \geq 2(n-3)\left(l^{+}-g^{+}\right)+2(n-1) \sum k_{i}+2 v+2 m^{+} \text {. }
$$

Summarizing the above two cases, from (3.4), we have

$$
\begin{aligned}
\text { Ind } D_{u^{+}} \geq & 2(n-3)\left(l^{+}-g^{+}\right)+2(n-1) \sum k_{i}+2 v+2 m^{+}, \\
\text {Ind } D_{u^{-}} \leq & 2 C_{1}(A)+2(n-3)\left(g^{+}-l^{+}\right) \\
& +2(n-1)\left(v-\sum k_{i}\right)-2 v+2 m^{-} .
\end{aligned}
$$

Since $\alpha_{i}^{+}=0,1 \leq i \leq m$, if $m^{+}>0$, we have for any $\beta_{b} \in H^{*}(Z)$,

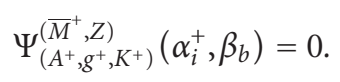

This implies $\Psi_{C}=0$ except $m^{-}=m$. So, we may assume $m^{-}=m$. We also may assume

$$
\sum \operatorname{deg} \alpha_{i}=2 C_{1}(A)+2 m
$$

Otherwise, Theorem 1.1 follows from the degree reason. Therefore, we have

$$
\begin{aligned}
\sum \operatorname{deg}\left(\alpha_{i}^{-}\right)= & 2 C_{1}(A)+2 m \\
> & 2 C_{1}(A)+2(n-3)\left(g^{+}-l^{+}\right) \\
& +2(n-1)\left(v-\sum k_{i}\right)-2 v+2 m^{-} \\
\geq & \operatorname{Ind} D_{u^{-}},
\end{aligned}
$$


since $g^{+} \leq g=1, v>0, k_{i}>0, n \geq 3$. Therefore, by the definition of relative GW invariants, we have for any $\beta_{b} \in H^{*}(Z)$,

$$
\Psi_{\left(A^{-}, g^{-}, K^{-}\right)}^{\left(\bar{M}^{-}, Z\right)}\left(\alpha_{i}^{-}, \beta_{b}\right)=0 .
$$

Therefore, $\Psi_{C}=0$ except $C=\left\{A^{-}, 1, m\right\}$.

So, now it remains to show that

$$
\Psi_{(p !(A), 1)}^{\tilde{M}}\left(p^{*} \alpha_{1}, \ldots, p^{*} \alpha_{m}\right)=\Psi_{\left(A^{-}, 1, m\right)}^{\left(\bar{M}^{-}, Z\right)}\left(\alpha_{1}^{-}, \ldots, \alpha_{m}^{-}\right) .
$$

To prove this, we perform the symplectic cutting for $\tilde{M}$ around the exceptional divisor $E$. Therefore, we have

$$
\overline{\tilde{M}}^{+}=\mathbf{P}\left(N_{E} \oplus 0\right), \quad \bar{M}^{-} \cong \tilde{M} .
$$

Now, we use the gluing formula to prove the contribution of stable $J$-holomorphic curves in $\tilde{M}$ which touch the exceptional divisor $E$ to the GW invariants of $\tilde{M}$ is zero. We consider the gluing component

$$
C=\left\{p !(A)^{+}, g^{+}, K^{+} ; p !(A)^{-}, g^{-}, K^{-}\right\}
$$

Since $\alpha_{i}^{+}=0,1 \leq i \leq m$, we have $\Psi_{C}=0$ except

$$
K^{+}=\left(k_{1}, \ldots, k_{v}\right), \quad K^{-}=(\underbrace{0, \ldots, 0}_{m}, k_{1}, \ldots, k_{v}) .
$$

From Proposition 2.1, we have (3.3), where $C_{1}$ denotes the first Chern class of $M$.

We assume that $u^{ \pm}: \Sigma^{ \pm} \rightarrow M^{ \pm}$has $l^{ \pm}$connected components $u_{i}^{ \pm}: \Sigma_{i}^{ \pm} \rightarrow M^{ \pm}, i=1, \ldots$, $l^{ \pm}$. From [5, Remark 2.3], it is not difficult to see that $\bar{u}_{i}^{+}$can be identified as stable $J$ holomorphic curve $h_{i}^{+}$in $\bar{M}$. Then, from [5, Proposition 2.4], we have

$$
\operatorname{Ind} D_{u^{+}}=\sum_{i=1}^{l^{+}} \operatorname{Ind} D_{\bar{u}_{i}^{+}}=(2 n-6) l^{+}+2 \sum_{i=1}^{l^{+}} C_{1}\left[h_{i}^{+}\right]+2 v-2 \sum k_{i} \text {, }
$$

where $C_{1}$ is the first Chern class of $\overline{\tilde{M}}^{+}$.

Let $V$ be a complex rank $r$ vector bundle over $X$, and $\pi: \mathbf{P}(V) \rightarrow X$ the corresponding projective bundle. Let $\xi_{V}$ be the first Chern class of the tautological line bundle in $\mathbf{P}(V)$. A simple calculation shows that

$$
C_{1}(\mathbf{P}(V))=\pi^{*} C_{1}(X)+\pi^{*} C_{1}(V)-r \xi_{V}
$$

Note that $\tilde{M}^{+}=\mathbf{P}\left(N_{E} \oplus 0\right)$ and $E=\mathbf{P}\left(N_{C}\right)$. Applying (3.18) to $\overline{\tilde{M}}^{+}$and $E$, we obtain

$$
\begin{aligned}
C_{1}\left(\overline{\tilde{M}}^{+}\right) & =C_{1}(E)+C_{1}\left(N_{E}\right)-2 \xi \\
& =C_{1}(C)+C_{1}\left(N_{C}\right)-(n-1) \xi_{1}+C_{1}\left(N_{E}\right)+2 \xi,
\end{aligned}
$$


where $\xi_{1}$ and $\xi$ are the first Chern classes of the tautological line bundles in $\mathbf{P}\left(N_{C}\right)$ and $\mathbf{P}\left(N_{E} \oplus 0\right)$, respectively. Here, we denote Chern class and its pullback by the same symbol. It is well known that the normal bundle to $E$ in $\tilde{M}$ is just the tautological bundle on $E \cong \mathbf{P}\left(N_{C}\right)$. Therefore, $C_{1}\left(N_{E}\right)=\xi_{1}$. So, we have

$$
C_{1}\left(\overline{\tilde{M}}^{+}\right)=C_{1}(M)-(n-2) \xi_{1}-2 \xi
$$

We know that $\bar{M}$ is a projective bundle over $E$ with fiber $\mathbf{P}^{1}$. Let $L$ be the class of a line in the fiber $\mathbf{P}^{1}$ and $e$ the class of a line in the fiber $\mathbf{P}^{n-2}$ in $E=\mathbf{P}\left(N_{C}\right)$. Denote by $\left[h_{i}^{+}\right]^{C}$ the homology class of the projection in $C$ of the curve $h_{i}^{+}$. Denote by $\left[h_{i}^{+}\right]^{F}$ the difference of $\left[h_{i}^{+}\right]$and $\left[h_{i}^{+}\right]^{F}$, that is, $\left[h_{i}^{+}\right]^{F}=\left[h_{i}^{+}\right]-\left[h_{i}^{+}\right]^{C}$. Then, it is easy to know $\left[h_{i}^{+}\right]^{F}=a L+b e$. Since $\xi \cdot\left[h_{i}^{+}\right]=\sum k_{j}$, where the summation runs over ends of $u_{i}^{+}$, and $E \cdot\left[h_{i}^{+}\right]=0$, so we have $\xi \cdot\left[h_{i}^{+}\right]^{F}=a=\sum k_{j}$ and $E \cdot\left[h_{i}^{+}\right]^{F}=a-b=0$. Therefore, we have $a=b=\sum k_{j}$. So, we have $\left[h_{i}^{+}\right]^{F}=\sum k_{j}(L+e)$. For Case 1 , we have $\left[h_{i}^{+}\right]^{C}=0$. Therefore, we have

$$
\sum_{i=1}^{l^{+}} C_{1}\left[h_{i}^{+}\right]=2(n-1) \sum k_{i}
$$

For Case 2, since $C_{1}(C)+C_{1}\left(N_{C}\right) \geq 0$, we have

$$
\sum_{i=1}^{l^{+}} C_{1}\left[h_{i}^{+}\right] \geq 2(n-1) \sum k_{i} .
$$

Plugging in (3.17), we have

$$
\text { Ind } D_{u^{+}} \geq 2(n-3)\left(l^{+}-g^{+}\right)+2(2 n-3) \sum k_{i}+2 \nu \text {. }
$$

Therefore,

$$
\begin{aligned}
\text { Ind } D_{u^{-}} \leq & 2 C_{1}(A)+2(n-3)\left(g^{+}-l^{+}\right) \\
& +(2 n-2)\left(v-\sum k_{i}\right)-2(n-2) \sum k_{i}+2 m .
\end{aligned}
$$

From the degree reason, we also may assume

$$
\sum \operatorname{deg}\left(p^{*} \alpha_{i}\right)=2 C_{1}(A)+2 m .
$$

Then,

$$
\begin{aligned}
\sum \operatorname{deg}\left(p^{*} \alpha_{i}\right)= & 2 C_{1}(A)+2 m \\
> & 2 C_{1}(A)+2(n-3)\left(g^{+}-l^{+}\right) \\
& +(2 n-2)\left(v-\sum k_{i}\right)-2(n-2) \sum k_{i}+2 m \\
\geq & \operatorname{Ind} D_{u^{-}}
\end{aligned}
$$


since $\nu>0, k_{i}>0$. Therefore, by the definition of relative GW invariants, we have for any $\beta_{b} \in H^{*}(Z)$,

$$
\Psi_{\left(p !(A)^{-}, 1, K^{-}\right)}^{\left(\overline{\bar{M}}^{-}, Z\right)}\left(\left(p^{*} \alpha_{i}\right)^{-}, \beta_{b}\right)=0 .
$$

Therefore, the contribution of $J$-holomorphic curves to the GW invariant is nonzero only if it does not touch the exceptional divisor $E$, that is, $C=\left\{p !(A)^{-}, 1, m\right\}$. Therefore, we have

$$
\Psi_{(p !(A), 1)}^{\tilde{M}}\left(p^{*} \alpha_{1}, \ldots, p^{*} \alpha_{m}\right)=\Psi_{\left(p !(A)^{-}, 1, m\right)}^{\left(\overline{\tilde{M}}^{-}, Z\right)}\left(\left(p^{*} \alpha_{1}\right)^{-}, \ldots,\left(p^{*} \alpha_{m}\right)^{-}\right) .
$$

Since $\bar{M}^{-}=\tilde{M}=\bar{M}^{-}$and $p !(A)$ is a nonexceptional class, we may identify the homology class $p !(A)^{-}$with $A^{-}$. Hence, Theorem 1.1 follows.

Proof of Theorem 1.2. Since $S$ is a smooth surface, the normal bundle $N_{S}$ is a symplectic vector bundle. By symplectic neighborhood theorem, there is a tubular neighborhood $\mathcal{N}_{\delta}(S)$ of $S$ which is symplectomorphic to the normal bundle $N_{S}$. We perform the symplectic cutting as in [5, Section 2.1]. We obtain

$$
\bar{M}^{+}=\mathbf{P}\left(N_{S} \oplus \mathcal{O}\right), \quad \bar{M}^{-}=\tilde{M} .
$$

We may assume $\alpha_{i}^{+}=0$ if we choose a sufficiently small $\delta>0$ because of the assumption of $\alpha_{i}$.

Similar to the proof of Theorem 1.1, we first consider the contribution of each gluing component to the GW-invariants. Therefore, we consider the component (3.2). From Proposition 2.1, we have (3.3).

We assume $u^{ \pm}: \Sigma^{ \pm} \rightarrow \bar{M}^{ \pm}$has $l^{ \pm}$connected components $u_{i}^{ \pm}: \Sigma_{i}^{ \pm} \rightarrow \bar{M}^{ \pm}, i=1, \ldots, l^{ \pm}$. Suppose $\Sigma_{i}^{ \pm}$has arithmetic genus $g_{i}^{ \pm}, g^{ \pm}=\sum g_{i}^{ \pm}$with $m^{ \pm}$marked points and $m^{ \pm}=\sum m_{i}^{ \pm}$.

The similar calculation to that in the proof of Theorem 1.1 shows that

$$
\operatorname{Ind} D_{u^{+}}=2(n-3)\left(l^{+}-g^{+}\right)+2(n-2) \sum k_{i}+2 v+2 m^{+} \text {. }
$$

Therefore, we have

$$
\text { Ind } D_{u^{-}}=2 C_{1}(A)+2(n-3)\left(g^{+}-l^{+}\right)+2(n-2)\left(v-\sum k_{i}\right)+2 m^{-} \text {. }
$$

The same argument as in the proof of Theorem 1.1 shows that the contribution of the component $C$ to the GW-invariant of $M$ is nonzero only if $C$ has (3.16). We also assume

$$
\sum \operatorname{deg} \alpha_{i}=2 C_{1}(A)+2 m
$$

The same argument as in the proof of Theorem 1.1 shows that there is a symplectic cutting such that $\Psi_{C}=0$ except $C=\left\{A^{-}, 1, m\right\}$. From the gluing theorem, we have

$$
\Psi_{(A, 1)}^{M}\left(\alpha_{1}, \ldots, \alpha_{m}\right)=\Psi_{\left(A^{-}, 1, m\right)}^{\left(\bar{M}^{-}, Z\right)}\left(\alpha_{1}^{-}, \ldots, \alpha_{m}^{-}\right)
$$


Now, it remains to prove

$$
\Psi_{(p !(A), 1)}^{\tilde{M}}\left(p^{*} \alpha_{1}, \ldots, p^{*} \alpha_{m}\right)=\Psi_{\left(A^{-}, 1, m\right)}^{\left(\bar{M}^{-}, Z\right)}\left(\alpha_{1}^{-}, \ldots, \alpha_{m}^{-}\right) .
$$

To prove this, we perform the symplectic cutting for $\tilde{M}$ around $E$ as in the proof of Theorem 1.1. Therefore, we have (3.14).

We also use the gluing theorem to prove that the contribution of stable $J$-holomorphic curves in $\tilde{M}$ which touch the exceptional divisor $E$ to the $\mathrm{GW}$ invariant of $\tilde{M}$ is zero. We consider the component (3.15).

Since $\alpha_{i}^{+}=0,1 \leq i \leq m$, we have $\Psi_{C}=0$ except (3.16). Similar calculation to that in the proof of Theorem 1.1 shows that

$$
\begin{aligned}
\text { Ind } D_{u^{+}}= & 2(n-3)\left(l^{+}-g^{+}\right)+2 v+2(2 n-5) \sum k_{i}, \\
\text { Ind } D_{u^{-}}= & 2 C_{1}(A)+2(n-3)\left(g^{+}-l^{+}\right) \\
& +2(n-2)\left(v-\sum k_{i}\right)-2(n-3) \sum k_{i}+2 m .
\end{aligned}
$$

The same argument as in the proof of Theorem 1.1 shows (3.28).

The rest of the proof is the same as that of the proof of Theorem 1.1. So, we omit it. This completes the proof of Theorem 1.2.

\section{Acknowledgments}

The first author would like to thank Professor Yongbin Ruan for his many suggestive discussions and encouragement. The first author also thanks Max-Planck-Institut für Mathematik in Bonn for providing an excellent research environment and support. This work was partially supported by NSF of China (1017114 and 10231050).

\section{References}

[1] M. Bershadsky, S. Cecotti, H. Ooguri, and C. Vafa, Holomorphic anomalies in topological field theories (with an appendix by S. Katz), Nuclear Phys. B 405 (1993), no. 2-3, 279-304.

[2] T. Eguchi, K. Hori, and C.-S. Xiong, Quantum cohomology and Virasoro algebra, Phys. Lett. B 402 (1997), no. 1-2, 71-80.

[3] E. Getzler, Intersection theory on $\bar{M}_{1,4}$ and elliptic Gromov-Witten invariants, J. Amer. Math. Soc. 10 (1997), no. 4, 973-998.

[4] P. Griffiths and J. Harris, Principles of Algebraic Geometry, Wiley-Interscience [John Wiley \& Sons], New York, 1978.

[5] J. Hu, Gromov-Witten invariants of blow-ups along points and curves, Math. Z. 233 (2000), no. 4, 709-739.

[6] Gromov-Witten invariants of blow-ups along surfaces, Compositio Math. 125 (2001), no. 3, 345-352.

[7] E.-N. Ionel and T. H. Parker, Gromov-Witten invariants of symplectic sums, Math. Res. Lett. 5 (1998), no. 5, 563-576.

[8] E. Lerman, Symplectic cuts, Math. Res. Lett. 2 (1995), no. 3, 247-258.

[9] A.-M. Li and Y. Ruan, Symplectic surgery and Gromov-Witten invariants of Calabi-Yau 3-folds, Invent. Math. 145 (2001), no. 1, 151-218.

[10] J. Li, A degeneration formula of $G W$-invariants, J. Differential Geom. 60 (2002), no. 2, 199-293. 
90 Elliptic GW invariants of blowups along curves and surfaces

[11] D. McDuff, Blow ups and symplectic embeddings in dimension 4, Topology 30 (1991), no. 3, 409-421.

[12] R. Pandharipande, A geometric construction of Getzler's elliptic relation, Math. Ann. 313 (1999), no. 4, 715-729.

[13] Y. Ruan, Topological sigma model and Donaldson-type invariants in Gromov theory, Duke Math. J. 83 (1996), no. 2, 461-500.

[14]_, Surgery, quantum cohomology and birational geometry, Northern California Symplectic Geometry Seminar (Y. Eliashberg, D. Fuchs, T. Ratiu, and A. Weinstein, eds.), Amer. Math. Soc. Transl. Ser. 2, vol. 196, American Mathematical Society, Rhode Island, 1999, pp. 183-198.

[15] Y. Ruan and G. Tian, A mathematical theory of quantum cohomology, J. Differential Geom. 42 (1995), no. 2, 259-367.

Jianxun Hu: Department of Mathematics, Zhongshan University, Guangzhou 510275, China E-mail address: stsjxhu@zsu.edu.cn

Hou-Yang Zhang: Department of Mathematics, The Science College, Beijing Jiaotong University, Beijing 100044, China

E-mail address: lpshao@center.njtu.edu.cn 


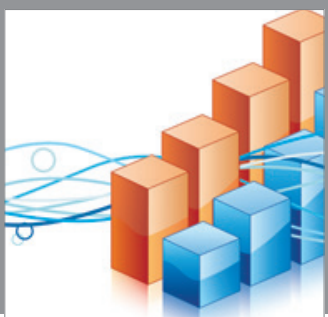

Advances in

Operations Research

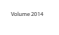

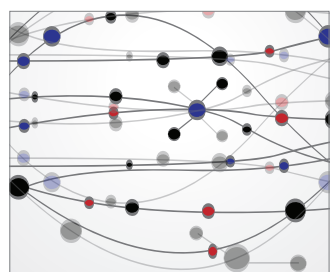

\section{The Scientific} World Journal
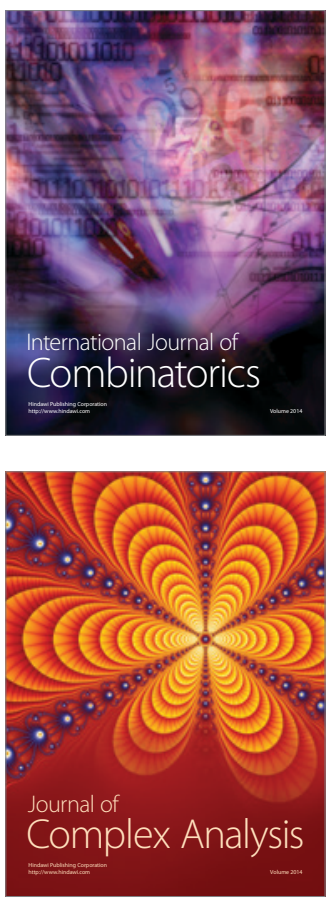

International Journal of

Mathematics and

Mathematical

Sciences
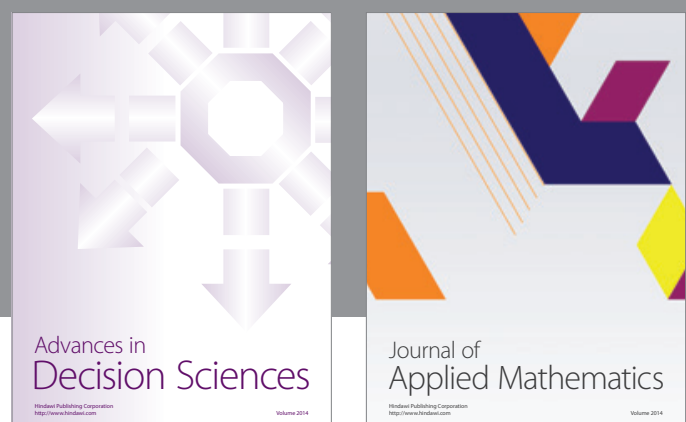

Journal of

Applied Mathematics
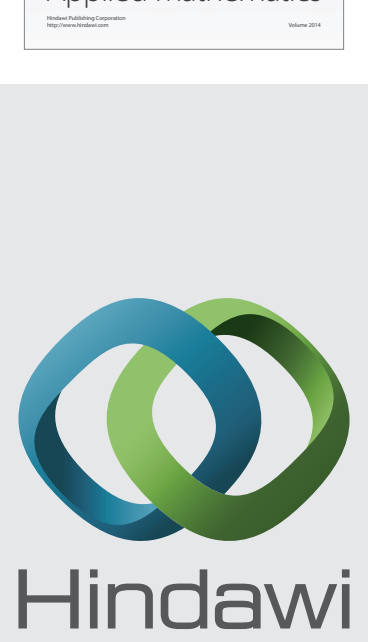

Submit your manuscripts at http://www.hindawi.com
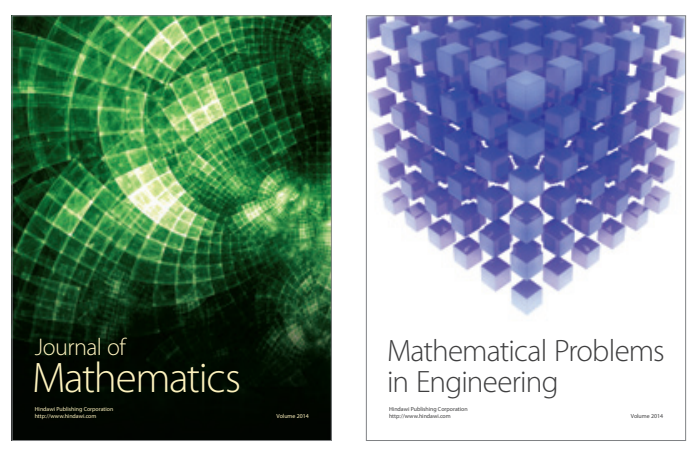

Mathematical Problems in Engineering
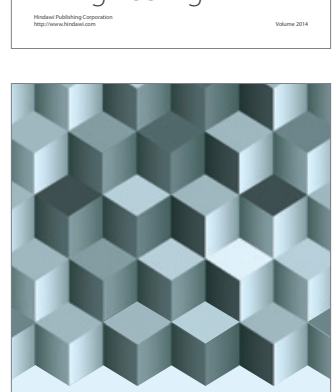

Journal of

Function Spaces
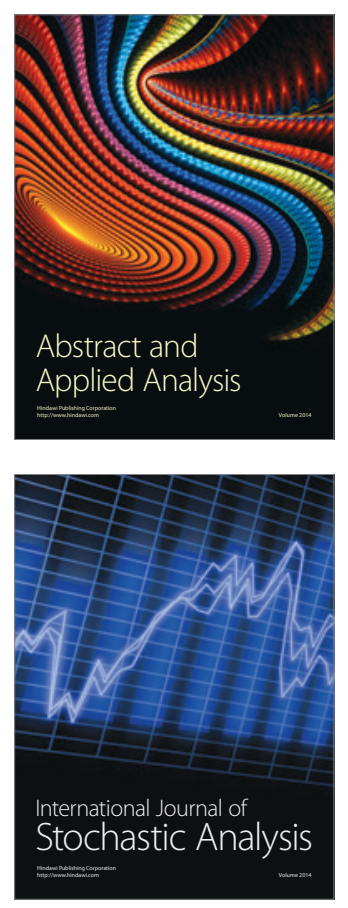

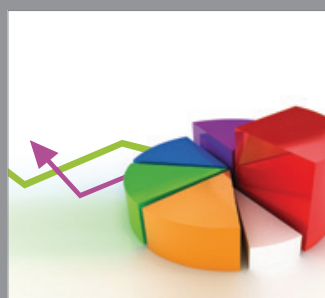

ournal of

Probability and Statistics

Promensencen
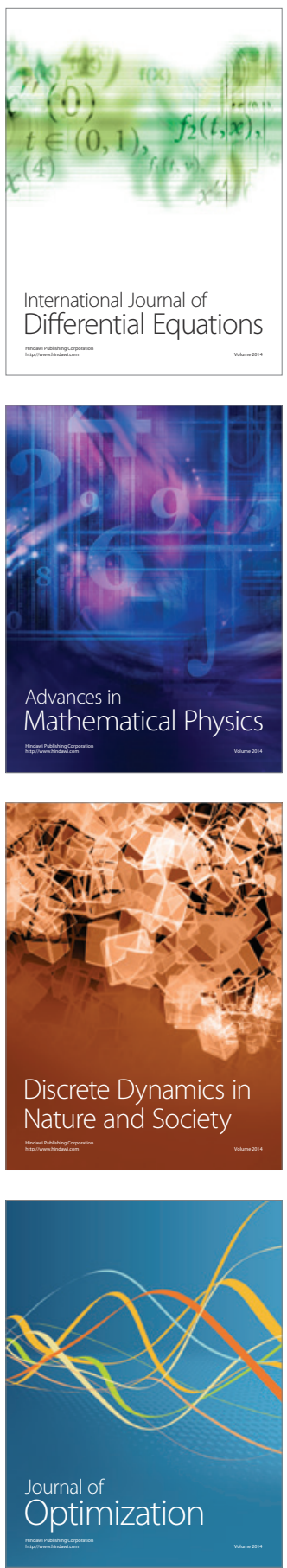